\title{
J oint Blind Parameter Estimation of Non-cooperative High-Order Modulated PCMA Signals
}

\author{
Yiming Guo ${ }^{1}$, Hua Peng ${ }^{1}$ and Jun Fu ${ }^{1}$ \\ ${ }^{1}$ China National Digital Switching System Engineering and Technological Research Center \\ Zhengzhou, Henan 450001 - China \\ [e-mail:guoym28892@163.com] \\ *Corresponding author: Yiming Guo
}

Received February 27, 2018; revised April 25, 2018; accepted May 15, 2018;

published October 30, 2018

\begin{abstract}
A joint blind parameter estimation algorithm based on minimum channel stability function aimed at the non-cooperative high-order modulated paired carrier multiple access (PCMA) signals is proposed. The method, which uses hierarchical search to estimate time delay, amplitude and frequency offset and the estimation of phase offset, including finite ambiguity, is presented simultaneously based on the derivation of the channel stability function. In this work, the structure of hierarchical iterative processing is used to enhance the performance of the algorithm, and the improved algorithm is used to reduce complexity. Compared with existing data-aided algorithms, this algorithm does not require a priori information. Therefore, it has significant advantage in solving the problem of blind parameter estimation of non-cooperative high-order modulated PCMA signals. Simulation results show the performance of the proposed algorithm is similar to the modified Cramer-Rao bound (MCRB) when the signal-to-noise ratio is larger than $16 \mathrm{~dB}$. The simulation results also verify the practicality of the proposed algorithm.
\end{abstract}

Keywords: Co-frequency detection, PCMA, non-cooperative, parameters estimation, joint blind estimation

This research was supported by the National Natural ScienceFoundation of China(No.61401511) and the National Natural ScienceFoundation of China(No.U1736107) 


\section{Introduction}

Paired carrier multiple access (PCMA) is a technology used to improve the capacity of satellite communication, which has been widely used in recent years [1]. Communication signals sent by two ground stations are overlapped in time and frequency domains. In the system of cooperative PCMA communication, each ground station sends an uplink signal and receives a downlink mixed signal of the local signal and the signal of the other ground stations at the same time. In the system of non-cooperative PCMA communication, the third-party receiver cannot use the cooperative communication method directly to obtain the useful signal component without any a priori information of both parties. Therefore, the downlink mixed signal contains the fixed mixed mode of the two uplink signal components. The positive definite condition required by the normal signal blind separation cannot be constructed. Thus, obtaining similar effects with the traditional multi-channel reception is impossible despite the use of multiple receive terminals on the ground. Therefore, the interference suppression technology based on the multi-channel reception is no longer applicable [2], and only the single-channel reception blind demodulation method [3-4] can be used.

Before symbol sequence demodulation, the modulation parameters and channel response of each signal component should be estimated. The key issue is how to estimate the frequency offset, time delay, amplitude and phase offset.

Parameter estimation of non-cooperative PCMA signal can be divided into data-aided and non-data-aided algorithms. Data-aided algorithms rely on the signal frame format, which can estimate the parameters depending on the correlation of frame synchronisation header data [5-7]. However, these algorithms are not applicable in blind signal processing. In non-data-aided algorithms, the modulated-type PCMA signal is utilised in low-order modulated signal at first, which estimates the frequency offset by extracting discrete spectral lines of square spectrum and fourth-power spectrum [8-10]. However, numerous cross terms are found when the high-order spectrums and the statistics of the high-order modulated (such as 8PSK) signals are calculated. In estimation algorithms of time delay, the technique of interference blind separation based on particle filter can estimate delays of the two signal components simultaneously [11-12], but it has high complexity and is sensitive to frequency offset. A joint time-delay estimation method based on the maximum-likelihood algorithm is proposed [13]. This method is not sensitive to frequency offset and has lower complexity than the particle filtering method. However, a large number of approximations of the cross terms exist in the derivation process. Therefore, the method is not suitable for high-order modulated PCMA signal. Compared with the diversity of time delay estimation algorithms, amplitude estimation and phase offset estimation algorithms focus mainly on high-power methods [14], but the problem of having too many cross terms produced in high-order modulated PCMA signals still exists. Furthermore, a priori information on frequency offset must be known. In summary, in current situations in which high-order modulated PCMA signals usually appear[15], the blind parameter estimation is an urgent problem that needs to be solved.

This work is devoted to solving the problem of joint parameter estimation of high-order modulated PCMA signal in non-data-aided algorithms. With the aid of per-survivor processing (PSP) algorithm [16], channel stability function is defined. First, the method of joint hierarchically searching parameters is used. Then, channel parameters are estimated by searching the values of channel parameters that causes the channel stability function to reach 
the minimum value. Blind estimation of all parameters is realised, and the cross terms are avoided at the same time.

\section{English Abbreviations}

Main notations used in this paper:

$h_{i}, f_{i}, \theta_{i}$ and $\tau_{i}(i=1,2)$ are the amplitudes, frequency offsets, initial phase offsets and time delays, respectively.

$\zeta_{i}$ : Phase value of $i$-th signal component.

$\hat{X}$ : Estimation value of $X$.

$\boldsymbol{g}_{i}(\cdot)$ : Pulse response of the channel filter.

$\boldsymbol{s}_{k}$ : State grid at time $k$.

$\lambda\left(s_{k-1} \rightarrow s_{k}\right)=\left|e\left(s_{k-1} \rightarrow s_{k}\right)\right|^{2}$ : Branch path metric at time $k$.

$\boldsymbol{s}_{k}^{m}\left(m=1,2 \cdots M^{4 L_{1}}\right)$ : Possible state at time $k$.

$\left(s_{k-1}^{\phi_{k+L_{1}}} \mid \boldsymbol{s}_{k}^{m}\right)$ : All states at time $(k-1)$ that can reach state $\boldsymbol{s}_{k}^{m}$ when the input symbol pair is $\phi_{k+L_{1}}=\left(\hat{a}_{1, k+L_{1}}, \hat{a}_{2, k+L_{1}}\right)$ at time $k$.

$\Gamma\left(s_{k}^{m}\right)$ : Accumulated path metric of $s_{k}^{m}$.

$\hat{\boldsymbol{G}}_{i, k}^{\mathrm{T}}\left(\boldsymbol{s}_{k}^{m}\right)$ : Channel response estimation of the $i$-th signal component in time $k$ at possible state $s_{k}^{m}$.

$\eta_{i, k}\left(s_{k}^{m}\right)$ : Channel stability function corresponding to the surviving path of state $s_{k}^{m}$ at time $k$.

\section{Signal Model}

In the system of non-cooperative PCMA communication, the ground station receives the mixed signal of two MPSK- or QAM-modulated signal components. The received signal is sampled at symbol period $T_{s}$, and its discrete-time form can be written as

$$
y_{k}=h_{1} e^{j\left(2 \pi f_{1} k T_{s}+\theta_{1}\right)} x_{1, k}+h_{2} e^{j\left(2 \pi f_{2} k T_{s}+\theta_{2}\right)} x_{2, k}+v_{k},
$$

where $v_{k}(k=1,2, \ldots)$ is the complex additive white Gaussian noise sampled sequences with zero mean and variance $\sigma^{2} \cdot x_{1, k}$ and $x_{2, k}$ are the mixed signal components. The modulated mode of the two components is assumed to be the same and independent of each other. Then, $x_{i, k}$ can be expressed as

$$
x_{i, k}=\sum_{m=-L_{1}}^{L_{1}} a_{i, m} g_{i}\left(k T_{s}-m T_{s}+\tau_{i}\right),
$$

where $h_{i}, f_{i}, \theta_{i}$ and $\tau_{i}(i=1,2)$ are the amplitudes, frequency offsets, initial phase offsets and time delays, respectively. The phase value $\zeta_{i}=2 \pi f_{i} k T_{s}+\theta_{i}$ is defined. $a_{1, k}$ and $a_{2, k}$ are the two transmission signal component sequences, whose values depend on the modulated mode. $\boldsymbol{g}_{i}($. is the pulse response of the channel filter (consisting of shaped and matched filters). Its effective range is $\left[-L_{1} T_{s}, L_{1} T_{s}\right], L_{1}$ is the number of filter trailing symbols. $\boldsymbol{G}_{i, k}=\left\{h_{i} e^{j\left(2 \pi f_{k} T_{s}+\theta_{i}\right)} g_{i}\left(k T_{s}+L_{1} T_{s}+\tau_{i}: k T_{s}-L_{1} T_{s}+\tau_{i}\right)\right\}, \boldsymbol{a}_{i, k}=\left\{a_{i, k-L_{1}: k+L_{1}}\right\}$. The received signal can be written as 


$$
y_{k}=\boldsymbol{G}_{1, k}^{\mathrm{T}} \boldsymbol{a}_{1, k}+\boldsymbol{G}_{2, k}^{\mathrm{T}} \boldsymbol{a}_{2, k}+v_{k} .
$$

The purpose of demodulation in signal receiver is to estimate the sequence of two symbol sequences $\left\{a_{1, k}, a_{2, k}, k=0,1, \cdots\right\}$ according to the received sequences $\left\{y_{k}, k=0,1, \cdots\right\}$, where the reconstructed channel $\boldsymbol{G}_{i, k}$ plays an important role. In this work, amplitude $h_{i}$, time delay $\tau_{i}$, phase value $\zeta_{i}$ (with ambiguity) and frequency offsets $f_{i}$ of the two signal components are estimated accurately to prepare for the reconstruction of the channel and separation process in back-end separation algorithm.

\section{Blind parameter estimation algorithm based on channel stability function}

Parameter estimation of the PCMA signal can be combined with the blind separation process of mixed signals. First, a large number of channel responses can be reconstructed through hierarchical traversing parameters (time delays, phase values and amplitudes of the two mixed signal components) in a definite range. Subsequently, a small amount of mixed signal data $(K<50)$ can be separated blindly with each reconstructed channel response. Second, the channel response is updated, and the channel stability function is calculated, as shown in (11). The more accurate the initial parameter value is set, the smaller the channel response update changes in the separation process and the smaller the corresponding channel stability function. Therefore, PCMA signal parameter estimation can be realised by searching the minimum channel stability function. The frequency offset of the baseband received signal is about $10^{-2} f_{d}$ order of magnitude, where $f_{d}$ is the symbol rate. So the phase value could be considered constantly because of the limited length of the mixed signal when estimating the time delay $\tau_{i}$, amplitude $h_{i}$ and phase value $\zeta_{i}$. Therefore, the algorithm is not affected by the frequency offset when estimating the parameters of time delay, amplitude and phase value. The frequency offsets of the two mixed signal components are estimated through the same method based on the estimation of time delays, amplitudes and phase values, except that a larger amount of mixed signal data $(K>50)$ would be utilised in a blindly separated process.

The rest of this section is organised as follows. Section 4.1 describes the basic PCMA signal blind separation method. Section 4.2 derives the channel stability function based on the iterative PSP algorithm and describes the parameter estimation algorithm based on channel stability function. Section 4.3 focuses on the algorithm improvement. Section 4.4 discusses the complexity of this method. The algorithm flow chart is shown in Fig. 1.

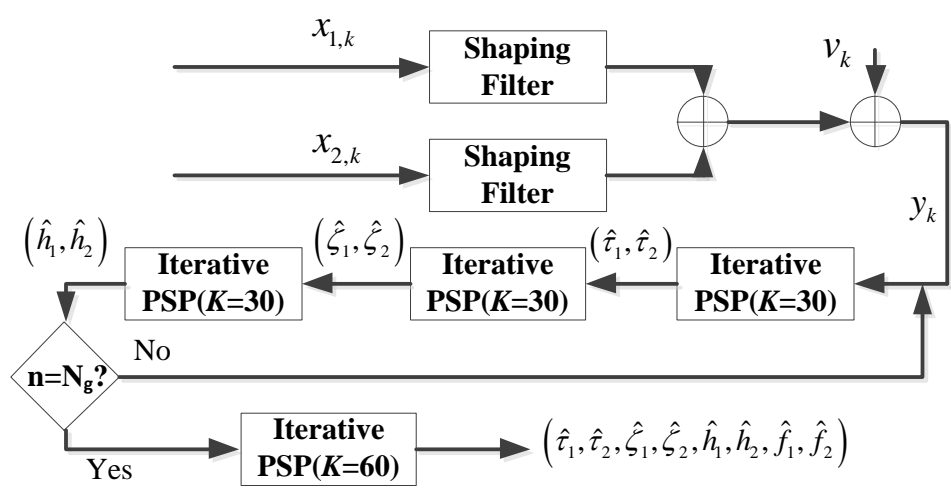

Fig. 1. Algorithm flow chartin this work 


\subsection{Blind separation of PCMA signal}

The maximum likelihood estimation of the transmitted symbol sequence is realised by searching the transmitted sequence $\boldsymbol{\Phi}$ that maximises the probability of receiving sequence $\boldsymbol{Y}$ in the joint space composed of $\boldsymbol{\Phi}$ and channel response $\boldsymbol{G}$ :

$$
\boldsymbol{\Phi}=\arg \max p(\boldsymbol{Y} \mid \boldsymbol{\Phi}, \boldsymbol{G}) .
$$

First, the state transition grid is constructed by taking the PSP algorithm as an example. The state grid at time $k-1$ is defined as $\boldsymbol{s}_{k-1}=\left[\hat{a}_{1, k-L_{1}: k+L_{1}-1}, \hat{a}_{2, k-L_{1}: k+L_{1}-1}\right]$. The input symbol pair of the two signal components and output symbol at time $k$ are defined as $\left(\hat{a}_{1, k+L_{1}}, \hat{a}_{2, k+L_{1}}\right)$ and $y_{k}$, respectively. $\hat{a}_{i, k}$ represents the estimation of $a_{i, k}$. If the state grid moves from $s_{k-1}$ to $s_{k}$, where $\boldsymbol{s}_{k}=\left[\hat{a}_{1, k-L_{1}+1: k+L_{1}}, \hat{a}_{2, k-L_{1}+1: k+L_{1}}\right]$, then the state transition process is as shown below:

$$
\boldsymbol{S}_{k-1} \underset{\hat{a}_{1, k+L_{1}}, \hat{a}_{2, k+L_{1}}}{\longrightarrow} \boldsymbol{S}_{k} .
$$

With the influence of Gaussian white noise, likelihood function (4) at time $K$ could be written as

$$
\begin{aligned}
p(\boldsymbol{Y} \mid \boldsymbol{\Phi}, \boldsymbol{G})= & p\left(\boldsymbol{y}_{0: K} \mid \hat{a}_{1,0: K+L_{1}}, \hat{a}_{2,0: K+L_{1}}, \hat{\boldsymbol{G}}_{1,0: K}, \hat{\boldsymbol{G}}_{2,0: K}\right) \\
& =\prod_{k=0}^{K} p\left(y_{k} \mid \hat{\boldsymbol{a}}_{1, k}, \hat{\boldsymbol{a}}_{2, k}, \hat{\boldsymbol{G}}_{1, k}, \hat{\boldsymbol{G}}_{2, k}\right) \\
& =F \cdot \exp \left(-\frac{1}{2 \sigma^{2}} \sum_{k=0}^{K}\left|\boldsymbol{y}_{k}-\hat{\boldsymbol{G}}_{1, k}^{\mathrm{T}} \hat{\boldsymbol{a}}_{1, k}-\hat{\boldsymbol{G}}_{2, k}^{\mathrm{T}} \hat{\boldsymbol{a}}_{2, k}\right|^{2}\right)
\end{aligned}
$$

where $F=\left(\frac{1}{\sqrt{2 \pi} \sigma}\right)$ and $\hat{\boldsymbol{G}}$ is the estimation of the real channel $\boldsymbol{G}$. According to (5), the branch path metric at time $k$ could be defined as

$$
\begin{gathered}
\lambda\left(\boldsymbol{s}_{k-1} \rightarrow \boldsymbol{s}_{k}\right)=\left|e\left(\boldsymbol{s}_{k-1} \rightarrow \boldsymbol{s}_{k}\right)\right|^{2}, \\
e\left(\boldsymbol{s}_{k-1} \rightarrow \boldsymbol{s}_{k}\right)=\boldsymbol{y}_{k}-\hat{\boldsymbol{G}}_{1, k}^{\mathrm{T}} \hat{\boldsymbol{k}}_{1, k}\left(\boldsymbol{s}_{k-1} \rightarrow \boldsymbol{s}_{k}\right)-\hat{\boldsymbol{G}}_{2, k}^{\mathrm{T}} \hat{\boldsymbol{a}}_{2, k}\left(\boldsymbol{s}_{k-1} \rightarrow \boldsymbol{s}_{k}\right),
\end{gathered}
$$

where $\hat{\boldsymbol{a}}_{i, k}\left(s_{k-1} \rightarrow s_{k}\right)$ is the symbol vector of the $i$ th channel signal corresponding to the grid state transition $\left(s_{k-1} \rightarrow s_{k}\right) . s_{k}^{m}\left(m=1,2 \cdots M^{4 L_{1}}\right)$ represents the possible state at time $k$, where $M$ is the modulation order. For each state $\boldsymbol{s}_{k}^{m}$, (8) is performed to obtain the accumulated path metric:

$$
\Gamma\left(\boldsymbol{s}_{k}^{m}\right)=\min _{\left(s_{k-1}^{g_{k} L_{1}} \mid s_{k}^{m}\right)}\left(\Gamma\left(\boldsymbol{s}_{k-1}^{\phi_{k+L_{1}}} \mid \boldsymbol{s}_{k}^{m}\right)+\lambda\left(\left(\boldsymbol{s}_{k-1}^{\phi_{k+L_{1}}} \mid \boldsymbol{s}_{k}^{m}\right) \rightarrow \boldsymbol{s}_{k}^{m}\right)\right),
$$

where $\left(\boldsymbol{s}_{k-1}^{\phi_{k+L_{1}}} \mid \boldsymbol{s}_{k}^{m}\right)$ represents all states at time $(k-1)$ that can reach state $\boldsymbol{s}_{k}^{m}$ if the input symbol pair is $\phi_{k+L_{1}}=\left(\hat{a}_{1, k+L_{1}}, \hat{a}_{2, k+L_{1}}\right)$ at time $k, \Gamma(s)$ represents the path metric of state $\boldsymbol{s}$ and $\left(s_{k-1}^{\phi^{\prime}} \mid s_{k}^{m}\right)$ represents the state at time $(k-1)$ that reaches the minimum value of (8). The channel response is estimated based on the corresponding symbol sequences of each surviving path.

$$
\hat{\boldsymbol{G}}_{i, k}^{\mathrm{T}}\left(\boldsymbol{s}_{k}^{m}\right)=\hat{\boldsymbol{G}}_{i, k-1}^{\mathrm{T}}\left(\boldsymbol{s}_{k-1}^{\phi^{\prime}} \mid \boldsymbol{s}_{k}^{m}\right)+\mu e\left(\left(\boldsymbol{s}_{k-1}^{\phi^{\prime}} \mid \boldsymbol{s}_{k}^{m}\right) \rightarrow \boldsymbol{s}_{k}^{m}\right) \hat{\boldsymbol{a}}_{i, k}\left(\left(\boldsymbol{s}_{k-1}^{\phi^{\prime}} \mid \boldsymbol{s}_{k}^{m}\right) \rightarrow \boldsymbol{s}_{k}^{m}\right),
$$


where $\mu$ is the step factor, whose convergence condition is $0<\mu<\frac{2}{\lambda_{\max }}$ [17]. $\lambda_{\max }$ is the maximum eigenvalue of the received signal autocorrelation matrix.Channel is initialised to $\hat{\boldsymbol{G}}_{i, 0}^{\mathrm{T}}$, which is determined by the initial time delay, phase value and amplitude value. Channel reconstruction $\hat{\boldsymbol{G}}_{i, k}^{\mathrm{T}}$ is used to calculate the branch path metric at time $k+1$.

\subsection{Parameter estimation algorithm based on channel stability function}

According to (9), we define the channel stability function corresponding to the surviving path of state $\boldsymbol{s}_{k}^{m}$ at time $k$ as

$$
\eta_{i, k}\left(\boldsymbol{s}_{k}^{m}\right)=\left\|\hat{\mathbf{G}}_{i, k}^{\mathrm{T}}\left(\boldsymbol{s}_{k}^{m}\right)-\hat{\mathbf{G}}_{i, k-1}^{\mathrm{T}}\left(\boldsymbol{s}_{k-1}^{\phi_{\min }} \mid \boldsymbol{s}_{k}^{m}\right)\right\|_{2} .
$$

The minimum cumulative metric state is defined as $s_{K}=\min _{m} \Gamma\left(s_{K}^{m}\right)$ at time $K$. Thus, the state reserved $s_{k}(k=K-1, K-2, \cdots, 1)$ at each time can be derived. In this case, the corresponding channel stability function of $K$ symbol pairs is defined as

$$
\eta_{K}=\sum_{i=1}^{2} \eta_{i, K}=\sum_{i=1}^{2} \sum_{k=1}^{K}\left\|\hat{\mathbf{G}}_{i, k}^{\mathrm{T}}\left(\mathbf{s}_{k}\right)-\hat{\mathbf{G}}_{i, k-1}^{\mathrm{T}}\left(\mathbf{s}_{k-1} \mid \mathbf{s}_{k}\right)\right\|_{2} .
$$

where $\|\mathbf{V}\|_{2}$ represents the 2-norm of vector $\mathbf{V}$. The channel response can be reconstructed for each set of initial time delay, amplitude and phase value parameters. The channel stability function $\eta_{K}$ at time $K$ can be obtained correspondingly. The parameters that minimise the channel stability function are selected as the final parameter estimation.

$$
\left\{\hat{\tau}_{i}, \hat{h}_{i}, \hat{\zeta}_{i}\right\}=\underset{\tau_{i}^{\prime}, h_{i}^{\prime}, \zeta_{i}^{\prime}}{\arg \min }\left\{\eta_{K} \mid \tau_{i}^{\prime}, h_{i}^{\prime}, \zeta_{i}^{\prime}\right\},
$$

where $\hat{\tau}_{i}$ and $\hat{h}_{i}$ are the estimation of the PCMA signal time delays and amplitudes, respectively, and $\hat{\zeta}_{i}$ is the estimation of the phase value. If frequency offsets $f_{i}$ are known, then $\hat{\theta}_{i}=\hat{\zeta}_{i}-2 \pi f_{i} k T_{s}$ represents the phase offset estimation. In MPSK-modulated situation, ambiguity on possessing integer multiple of $\frac{2 \pi}{M}$ in $\hat{\theta}_{i}$ relative to the true phase offset value will arise because of the symmetry of modulated phase, which requires the removal of other means (such as differential coding and synchronous code). The channel response is reconstructed for each set of initial frequency offset $f_{i}^{\prime}$ through the same method, and the corresponding channel stability function $\eta_{K}$ is obtained at time $K$. Parameter $f_{i}^{\prime}$ that minimises the channel stability function is selected as the final frequency offset estimation:

$$
\left\{\hat{f}_{i}\right\}=\underset{f_{i}^{\prime}}{\arg \min }\left\{\eta_{K} \mid f_{i}^{\prime}, \hat{\tau}_{i}, \hat{h}_{i}, \hat{\zeta}_{i}\right\} .
$$

In summary, joint estimation algorithm steps based on channel stability function are as follows.

Initialisation: Normalise the energy of received sequence. The channel amplitude ratio is initialised to 1:1 and phase values are initialised to zero. The complexity and performance of the algorithm are considered comprehensively to set the value of separation symbol length $K$ and algorithm iteration number $N_{g}$. 
For the $n$th iteration:

Step 1: The time delays $\left(\tau_{1}^{\prime}, \tau_{2}^{\prime}\right)$ are traversed hierarchically in a definite range. The channel response $\hat{\boldsymbol{G}}_{i, 0}^{\mathrm{T}}\left(\tau_{1}^{\prime}, \tau_{2}^{\prime}\right)$ is then reconstructed. The estimation of time delay $\left(\hat{\tau}_{1}, \hat{\tau}_{2}\right)$ is determined when the channel stability function $\left\{\eta_{K} \mid \tau_{1}^{\prime}, \tau_{2}^{\prime}\right\}$ according to (11) reaches the minimum value. The estimation result of time delays $\left(\hat{\tau}_{1}, \hat{\tau}_{2}\right)$ is carried to Step 2.

Step 2: The phase values $\left(\zeta_{1}^{\prime}, \zeta_{2}^{\prime}\right)$ are traversed hierarchically in a definite range. The channel response $\hat{\boldsymbol{G}}_{i, 0}^{\mathrm{T}}\left(\hat{\tau}_{1}, \hat{\tau}_{2}, \zeta_{1}^{\prime}, \zeta_{2}^{\prime}\right)$ is reconstructed. The estimation of phase value $\left(\hat{\zeta}_{1}, \hat{\zeta}_{2}\right)$ is determined when the channel stability function $\left\{\eta_{K} \mid \hat{\tau}_{1}, \hat{\tau}_{2}, \zeta_{1}^{\prime}, \zeta_{2}^{\prime}\right\}$ according to (11) reaches the minimum. The estimation result of phase values $\left(\zeta_{1}^{\prime}, \zeta_{2}^{\prime}\right)$ is carried to Step 3.

Step 3: The amplitudes $\left(h_{1}^{\prime}, h_{2}^{\prime}\right)$ are traversed hierarchically in a definite range. The channel response $\hat{\boldsymbol{G}}_{i, 0}^{\mathrm{T}}\left(\hat{\tau}_{1}, \hat{\tau}_{2}, \hat{\zeta}_{1}, \hat{\zeta}_{2}, h_{1}^{\prime}, h_{2}^{\prime}\right)$ is also reconstructed. The estimation of amplitude $\left(\hat{h}_{1}, \hat{h}_{2}\right)$ is determined when the channel stability function $\left\{\eta_{K} \mid \hat{\tau}_{1}, \hat{\tau}_{2}, \hat{\zeta}_{1}, \hat{\zeta}_{2}, h_{1}^{\prime}, h_{2}^{\prime}\right\}$ according to (11) reaches the minimum.

Step 4: $n=N_{g},\left\{\hat{\tau}_{1}, \hat{\tau}_{2}, \hat{\zeta}_{1}, \hat{\zeta}_{2}, \hat{h}_{1}, \hat{h}_{2}\right\}$ is the final result of the estimation of time delay, phase value and amplitude. The result is carried to Step 5. Otherwise, by setting $n=n+1$, the parameter value is initialised to $\left\{\hat{\tau}_{1}, \hat{\tau}_{2}, \hat{\zeta}_{1}, \hat{\zeta}_{2}, \hat{h}_{1}, \hat{h}_{2}\right\}$ and return to step 1 .

Step 5: The frequency offsets $\left(f_{1}^{\prime}, f_{2}^{\prime}\right)$ are traversed hierarchically in a definite range. Then, the channel response $\hat{\boldsymbol{G}}_{i, 0}^{\mathrm{T}}\left(f_{1}^{\prime}, f_{2}^{\prime}\right)$ is reconstructed. The estimation of frequency offsets $\left(\hat{f}_{1}, \hat{f}_{2}\right)$ is determined when the channel stability function $\left\{\eta_{K} \mid f_{i}^{\prime}, \hat{\tau}_{i}, \hat{h}_{i}, \hat{\zeta}_{i}\right\}$ according to (11) reaches the minimum value.

We take Step 1 as an example to describe the hierarchical traversing process. For the initialization of $m=1$ st staging process, the traversal spaces of $\tau_{1}^{\prime}$ and $\tau_{2}^{\prime}$ are $\left[-T_{s} / 2, T_{s} / 2\right] . \mu_{\tau}^{1}$ is the initial step size. $N_{e}$ is the number of hierarchical iterations. During the $m$-th staging process, $\frac{T_{s}}{\rho^{m-1} \mu_{\tau}^{m}} \cdot \frac{T_{s}}{\rho^{m-1} \mu_{\tau}^{m}}$ groups of delay parameters are traversed to search time delay estimation $\left(\hat{\tau}_{1}^{\prime}, \hat{\tau}_{2}^{\prime}\right)$ that enables channel stability function $\left\{\eta_{K} \mid \tau_{1}^{\prime}, \tau_{2}^{\prime}\right\}$ to reach the minimum value, where $\rho(\rho>1)$ is the shrinkage ratio. If $m=N_{e},\left(\hat{\tau}_{1}^{\prime}, \hat{\tau}_{2}^{\prime}\right)$ is determined as the result of time delay estimation. Otherwise $m=m+1$. The traversal spaces are set to $\left\{\left(\hat{\tau}_{1}^{\prime}-\frac{T_{s}}{2 \rho^{m-1}} \sim \hat{\tau}_{1}^{\prime}+\frac{T_{s}}{2 \rho^{m-1}}\right),\left(\hat{\tau}_{2}^{\prime}-\frac{T_{s}}{2 \rho^{m-1}} \sim \hat{\tau}_{2}^{\prime}+\frac{T_{s}}{2 \rho^{m-1}}\right)\right\}$. Step size is set to $\mu_{\tau}^{m+1}=\frac{\mu_{\tau}^{m}}{\rho}$, and the $m+1$-th hierarchical process is performed. The difference between Step 5 and the previous 
steps (Step 1, Step 2 and Step 3) is the selection of traversal step. The initialization of frequency offsets should be more accurate, that is, the value of $\mu_{f}^{1}$ in Step 5 should be smaller than $\mu_{\tau}^{1}$ in Step 1. Otherwise, the difference between the estimated phase and real phase values exhibits a periodic variation with the symbol separation process. Therefore, the frequency offsets are separately estimated in this study.

\subsection{Algorithm improvement}

The number of states that should be calculated for each symbol demodulation is complex for high-order modulated mixed signals. The number of reserved states at time $k$ is $M^{4 L_{1}}$, as described in Section 4.1. In this study, the $N_{\max }$-PSP algorithm is used to reduce the number of surviving paths [14]. The impossible path will be discarded to reduce the computation, and the maximum number of paths $N_{\max }$ will be retained based on a certain metric principle. If the number of surviving paths at state $\boldsymbol{s}_{k}$ is $L_{n}$, the number of extensive branch paths is $M^{2} L_{n}$ when the state changes from $s_{k}$ to $s_{k+1}$. If $M^{2} L_{n} \leq N_{\max }$, then $L_{n+1}=M^{2} L_{n}$. Otherwise, all paths are sorted based on the path metric, and the best $N_{\max }$ paths are kept, which update $L_{n+1}=N_{\max }$.

\subsection{Complexity analysis}

In this study, the parameter estimation algorithm is based on blind separation. The number of real multiplication and real addition is used as the complexity evaluation criteria. The computational complexity of the algorithm is derived mainly from the calculation of path metric in the separation algorithm and the tracking of channel response. In calculating the branch metric, complexity is concentrated mainly on the calculation of (8). $K M^{2\left(2 L_{1}+1\right)}\left(16 L_{1}+9\right)$ times of real addition and $K M^{2\left(2 L_{1}+1\right)}\left(16 L_{1}+10\right)$ times of real multiplication are required. In tracking the channel response, complexity is concentrated in (9) and (10). $2 K M^{2\left(2 L_{1}+1\right)}\left(24 L_{1}+11\right)$ times of real addition and $16 K M^{2\left(2 L_{1}+1\right)}\left(2 L_{1}+1\right)$ times of real multiplication are required. Thus, considering algorithmiteration number $N_{g}$ andhierarchical iteration $N_{e}$, the total times of real addition and multiplication are about $4 N_{g} N_{e} K M^{2\left(2 L_{1}+1\right)}\left(64 L_{1}+31\right)$ and $8 N_{g} N_{e} K M^{2\left(2 L_{1}+1\right)}\left(24 L_{1}+13\right)$, respectively. The total times of real addition and multiplication are reduced to about $4 N_{g} N_{e} K M^{2}\left(64 L_{1}+31\right) N_{\max }$ and $8 N_{g} N_{e} K M^{2}\left(24 L_{1}+13\right) N_{\max }$, respectively if we adopt the improved $N_{\max }$-PSP algorithm. The complexity of $N_{\max }-$ PSP algorithm is much lower than the unimproved algorithm. Table. 1 shows the complexity of the algorithm.

Table 1. Complexity of the Proposed Algorithm

\begin{tabular}{ccc}
\hline \hline Algorithm & Real additions & Real multiplications \\
\hline PSP algorithm & $4 N_{g} N_{e} K M^{2\left(2 L_{1}+1\right)}\left(64 L_{1}+31\right)$ & $8 N_{g} N_{e} K M^{2\left(2 L_{1}+1\right)}\left(24 L_{1}+13\right)$ \\
\hline$N_{\text {max }}$-PSP algorithm & $4 N_{g} N_{e} K M^{2}\left(64 L_{1}+31\right) N_{\max }$ & $8 N_{g} N_{e} K M^{2}\left(24 L_{1}+13\right) N_{\max }$ \\
\hline \hline
\end{tabular}

The performance of the algorithm reaches the stability function by using a small number of separation symbols (generally $K<60$ ). Under this condition, the complexity of the algorithm is maintained at a low level. Thus, this algorithm could be used in practical signal processing. 


\section{Simulation}

In this study, we use 8PSK modulated PCMA signals as an example to study the performance of the proposed algorithm. When $K=30$, the square root raised cosine FIR with a roll-off factor of 0.35 is employed in the shaping and matched filters of the two signals. The effective interval of ISI is [-3Ts, 3T $]$. Time delays $\tau_{1}$ and $\tau_{2}$, phase values $\theta_{1}$ and $\theta_{2}$, amplitudes $h_{1}$ and $h_{2}$, and frequency offsets $f_{1}$ and $f_{2}$ are selected randomly in space $\left[-T_{s} / 2\right.$, $\left.T_{s} / 2\right],[-\pi, \pi],[0.5,1.5]$ (the amplitude ratio of the energy-normalized mixed signal) and $\left[-10^{-2}, 10^{-2}\right]$ (relative to the symbol rate), respectively.The two-way signal delays, amplitudes and phase values are generated randomly.The values of the remaining simulation parameters are presented in Table. 2.

Table 2. Simulation Parameters

\begin{tabular}{c|c}
\hline \hline Initial step size & $\begin{array}{c}\mu_{\tau}^{1}=0.1 T_{s}, \mu_{\theta}^{1}=0.2 \pi, \mu_{h}^{1}=0.1 \text { and } \mu_{f}^{1} \\
=10^{-4} \text { (relative to symbol rate) }\end{array}$ \\
\hline Shrinkage ratio in $\{\tau, \theta, h\}$ estimation & $\rho_{\tau}=\rho_{\theta}=\rho_{h}=10$ \\
\hline Shrinkage ratio in $f$ estimation & $\rho_{f}=100$ \\
\hline$N_{\max }$ & 64 \\
\hline Hierarchical iteration number & $N_{e}=2$ \\
\hline Algorithm iteration number & $N_{g}=3$ \\
\hline Step size & $\mu=0.01$ \\
\hline \hline
\end{tabular}

The mean (normalized) estimated variance $\sigma_{e}^{2}$ is used to measure the parameter estimation performance, which is defined as the mean estimation variance of two signal components (normalized), that is, $\sigma_{e}^{2}=\left(\sigma_{\varphi_{1}}^{2}+\sigma_{\varphi_{2}}^{2}\right) / 2(\varphi \in\{\tau, h, f\})$ and $\sigma_{\varphi_{i}}^{2}=\left(\varphi_{i}-\hat{\varphi}_{i}\right)^{2}$.

\subsection{Modified Cramer-Rao Bound (MCRB) of parameter estimation}

We define parameter set $w=(\tau, h, f)^{\mathrm{T}}=\left(w_{1}, w_{2}, \cdots, w_{6}\right)^{\mathrm{T}}$, where $\boldsymbol{\tau}=\left(\tau_{1}, \tau_{2}\right)^{\mathrm{T}}, \boldsymbol{h}=\left(h_{1}, h_{2}\right)^{\mathrm{T}}$ and $\boldsymbol{f}=\left(f_{1}, f_{2}\right)^{\mathrm{T}}$. The received signal vector is denoted by $y$. When estimating the parameters $w$ jointly, CRB for the estimation of parameter $w_{m}(m=1,2, \cdots, 6)$ is a lower bound on the variance of any unbiased estimate, as shown in (14) and (15).

$$
\begin{gathered}
E_{\boldsymbol{y}}\left[\left(\hat{\boldsymbol{w}}_{m}-w_{m}\right)^{2}\right] \geq \operatorname{CRB}_{\boldsymbol{w}}\left(w_{m}\right)=\left[\mathbf{I}^{-1}(\boldsymbol{w})\right]_{m, m}, \\
{[\mathbf{I}(\boldsymbol{w})]_{m, n}=E_{\boldsymbol{y}}\left[-\frac{\partial^{2} \ln p(\boldsymbol{y} \mid \boldsymbol{w})}{\partial w_{m} \partial w_{n}}\right],}
\end{gathered}
$$

where $\mathrm{CRB}_{w}$ indicates $\mathrm{CRB}$ of the joint estimation of $\boldsymbol{w}, \mathbf{I}(\cdot)$ is the Fisher information matrix, $[\cdot] m, n$ denotes the matrix factor with row $m$ and column $n(n=1, \ldots, 6), E y[\cdot]$ denotes the statistical expectation of $\boldsymbol{y}$ and $p(\boldsymbol{y} \mid \boldsymbol{w})$ is the probability density function of $\boldsymbol{y}$ for a given $\boldsymbol{w}$. Accordingly, $\log$ likelihood function $\ln p(y \mid w)$ with an unknown $s$ can be expressed as follows:

$$
\ln p(\boldsymbol{y} \mid \boldsymbol{w})=\ln E_{s}[p(\boldsymbol{y} \mid \boldsymbol{w}, \boldsymbol{A})]=\ln \left(\sum_{j=0}^{M^{2 L}-1} \operatorname{Pr}\left[\boldsymbol{A}=\boldsymbol{c}_{j}\right] p\left(\boldsymbol{y} \mid \boldsymbol{w}, \boldsymbol{c}_{j}\right)\right),
$$

where $L=2 L_{1}+1, \boldsymbol{A}=\left\{\boldsymbol{a}_{1}, \boldsymbol{a}_{2}\right\}, p(\boldsymbol{y} \mid \boldsymbol{w}, \boldsymbol{A})$ is the probability density function of $\boldsymbol{y}$ for the given $\boldsymbol{w}$ and $\boldsymbol{A}$ and $E \boldsymbol{s}[\cdot]$ denotes the statistical expectation over $\boldsymbol{A}$. We assume that the data symbols are independent of one another regardless of whether they originate from the same or different sources. Hence, we obtain $\operatorname{Pr}\left[\boldsymbol{A}=\boldsymbol{c}_{j}\right]=M^{-2 L}$. High complexity is required to solve $\ln p(\boldsymbol{y} \mid \boldsymbol{w})$ for 
a large $L$. Therefore, MCRB is adopted to assess performance bound, which is slightly looser than the true $\mathrm{CRB}$, that is,

$$
\begin{aligned}
& \operatorname{CRB}_{\boldsymbol{w}}\left(w_{m}\right) \geq\left[\mathbf{I}^{-1}(\boldsymbol{w}, \boldsymbol{A})\right]_{m, m}=\operatorname{MCRB}_{\boldsymbol{w}}\left(w_{m}\right), \\
& {[\mathbf{I}(\boldsymbol{w}, \boldsymbol{A})]_{m, n}=E_{\boldsymbol{A}}\left\{E_{\boldsymbol{y} \mid \boldsymbol{A}}\left[-\frac{\partial^{2} \ln p(\boldsymbol{y} \mid \boldsymbol{w}, \boldsymbol{A})}{\partial w_{m} \partial w_{n}}\right]\right\} .}
\end{aligned}
$$

For a given $\boldsymbol{w}$ and $\boldsymbol{A}$ and corresponding receiving $\hat{\boldsymbol{y}}$, the ML function $p(\boldsymbol{y} \mid \boldsymbol{w}, \boldsymbol{A})$ attains a complex Gaussian distribution, as shown in the following expression:

$$
p(\boldsymbol{y} \mid \boldsymbol{w}, \boldsymbol{A})=\left(\pi N_{0}\right)^{-1 / 2} \exp \left(-\frac{1}{N_{0}} \int|y-\hat{y}|^{2} d t\right) .
$$

The following formula can be derived as follows [18]

$$
\begin{gathered}
\operatorname{MCRB}_{w}\left(\tau_{i}\right)=\frac{\left(1+\eta_{i}\right)}{2 L \dot{F}_{2}(0) / F_{2}(0) E_{s} / N_{0}}, \\
\operatorname{MCRB}_{h}\left(h_{i}\right)=\frac{N_{0}}{2 C L F_{2}(0)}, \\
\operatorname{MCRB}_{w}\left(f_{i}\right)=\frac{3\left(1+\eta_{i}\right)}{2 \pi^{2}\left(L^{2}-1\right) L T^{2} E_{s} / N_{0}},
\end{gathered}
$$

where $\eta_{i}=\left\{\begin{array}{c}\lambda^{2}, \quad i=1 \\ 1 / \lambda^{2}, i=2\end{array}, \lambda=h_{2} / h_{1}\right.$ and $F(\omega)$ is the Fourier transform of $G_{i}(t)$. $F_{2}(w)=F(w) \otimes F(w) / 2 \pi$, where $\otimes$ is the convolution operation, $\dot{F}_{2}(w)=d F_{2}(w) / d w$ and $E\left[a_{m}^{i_{1}}\left(a_{n}^{i_{2}}\right)^{*}\right]=\left\{\begin{array}{l}C \text { for } m=n \text { and } i_{1}=i_{2} \\ 0 \text { otherwise }\end{array}\right.$.

\subsection{Simulation performance of the algorithm}

The received signal is oversampled by 4 . The simulation results are compared with MCRB [18-20], with $N_{g}=3$ and $K=30$.

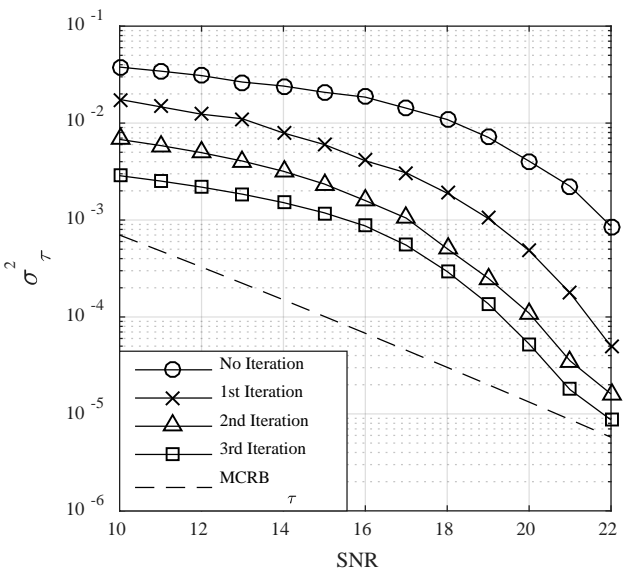

Fig. 2. Performance of time delay estimation

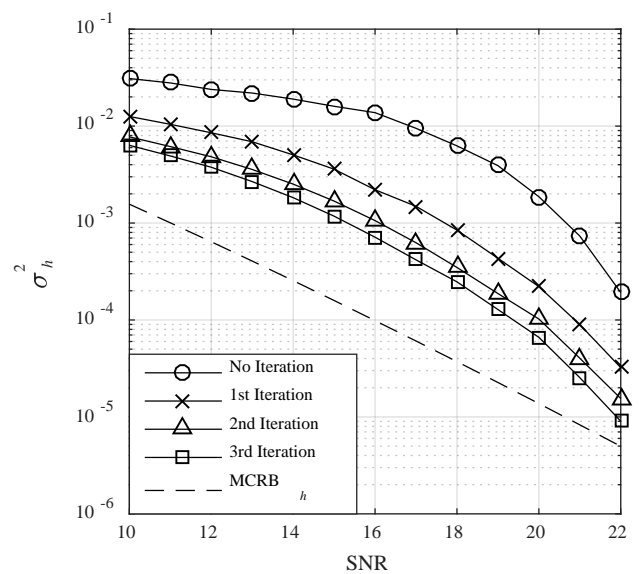

Fig. 3. Performance of amplitude estimation 
The curves of $\sigma_{\tau}^{2}$ and $\sigma_{h}^{2}$ versus the signal-to-noise ratio (SNR) in different iterations are shown in Figs. 2and 3, respectively. The time delay estimation variance rapidly approaches the bound above $18 \mathrm{~dB}$. Compared with no iteration in the $10^{-3}$ order of magnitude of $\sigma_{\tau}^{2}$, the algorithm obtains nearly $2 \mathrm{~dB}$ and nearly $3 \mathrm{~dB}$ SNR gain after the first and second iterations, respectively. At the same time, compared with no iteration in the $10^{-3}$ order of magnitude of $\sigma_{h}^{2}$, the algorithm obtains nearly $1.5 \mathrm{~dB}$ and nearly $2.5 \mathrm{~dB}$ SNR gain after the first and second iterations, respectively. These results can be explained as follows. The parameter values are initialized accurately with the increase in the number of iterations, and they provide considerable reliable prior information for the back-end separation algorithm. The channel stability function under the condition of correct parameters is highlighted, and the performance of the algorithm is improved. When the number of iterations is more than 2 , the accuracy of priori information is saturated to enhance the performance of the back-end separation algorithm. Thus, the improved space of performance is small. The selected number of iterations is twice considering the complexity of each iterative operation. The upper limit of iteration number $N_{g}$ in subsequent simulation is also set to 2 . The performance of frequency offset estimation is shown in Fig. 4, where $K=60$.

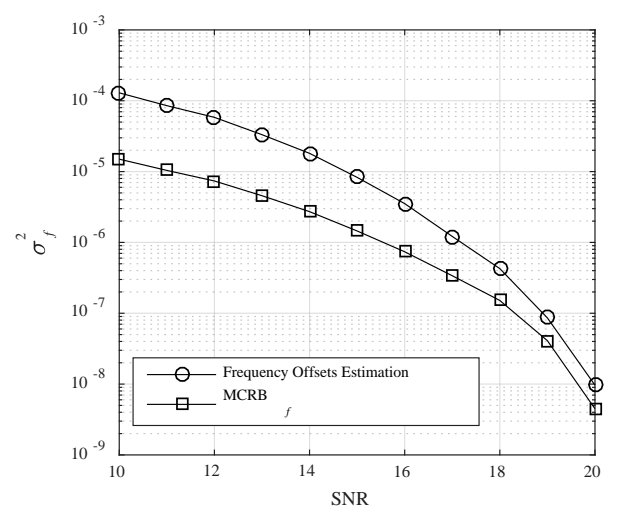

Fig. 4. Performance of frequency offset estimation

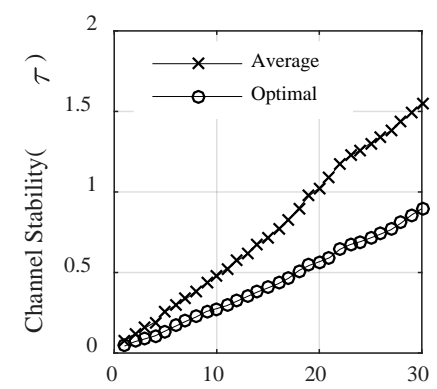

(a) $\mathrm{K}$

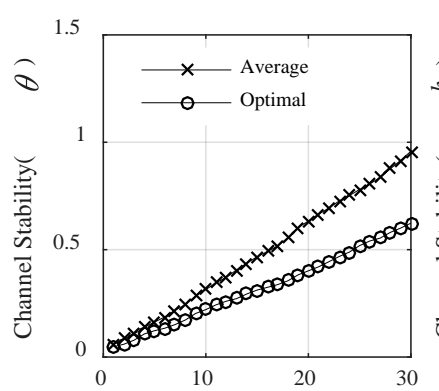

(b) $K$

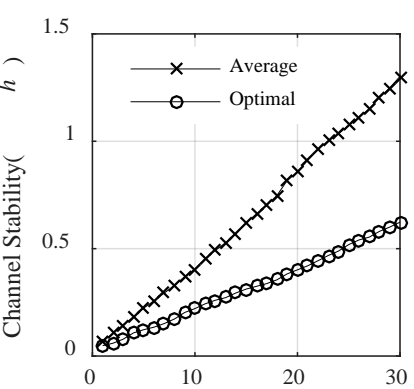

(c) $K$

Fig. 5. Channel stability $\left(N_{g}=0\right)$ 


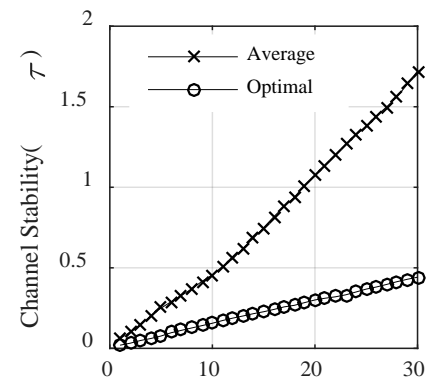

(a) $K$

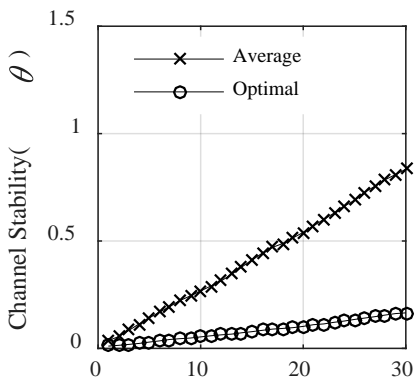

(b) $K$

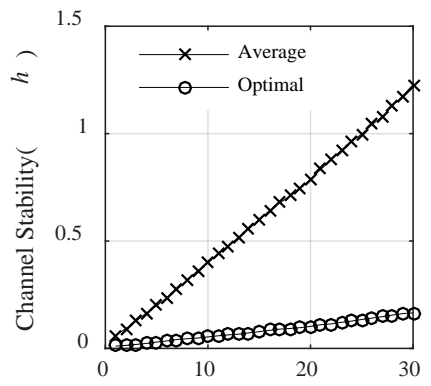

(c) $K$

Fig. 6. Channel stability $\left(N_{g}=2\right)$

The channel stability function curves versus the number of separated symbols are presented in Figs. 5 and 6, which represent no iteration and second iteration, respectively. The estimated target parameters in Figs. 5(a), 5(b) and 5(c) are time delays, phase offsets and amplitudes, respectively. On the basis of parameter setting, the number of tracking curves is 100 in each figure. "Average" represents the channel stability average curve for 100 experimental parameters, whereas "Optimal” represents the channel stability curve under optimal parameter condition. With an increase in the number of iterations, the gap between the "Optimal” curve and the "Average” widens, and a wide gap leads to a reliable result.

\subsection{Estimation performance of phase offset}

This method obtains the phase offsets with certain ambiguity. The initial phase values $\theta_{i}(i=1,2)$ of two signal components are generated randomly. Monte Carlo experiments are performed 30 times to estimate the mixed-signal phase offset. SNR $=14 \mathrm{~dB}, N_{g}=2$ and the remaining parameters are the same as those in Section 5.1.

The estimation results on the phase offsets of two-way signal components are shown in Figs. $7(\boldsymbol{a})$ and $\mathbf{7}(\boldsymbol{b}) . \theta_{i}(i=1,2)$ and $\hat{\theta}_{i}$ denote the true and estimated values, respectively. As shown in Fig. 7, the phase-offset estimation is obtained with the integer ambiguity of $\pi / 4$ for 8PSK modulation PCMA signals. This estimation is obtained from the symmetry of MPSK modulated signals, and should be removed by other means (such as differential coding and synchronization code). Therefore, the traversal space of phase isreduced to $\pi / 4$ in the algorithm to reduce complexity. If ambiguity is removed, the phase estimation performance of this algorithm is shown in Fig. 8 If signal frequency offset $f_{i}$ is not equal to zero, the phase value range of symbol sequence used in this method is $K f_{i}$, where $f_{i}<0.01$ (relative to symbol rate) in general. Thus, the phase values of $K$-symbols are constant. This condition is also the reason for theestimations on time delays and amplitudes not being affected by the error of frequency offsets. 


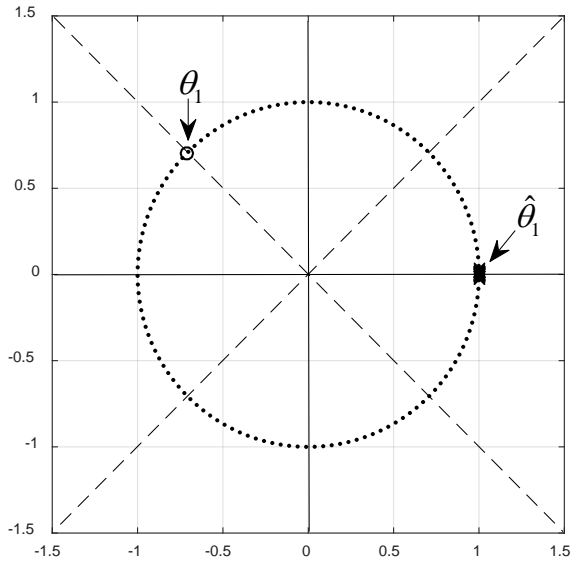

(a)

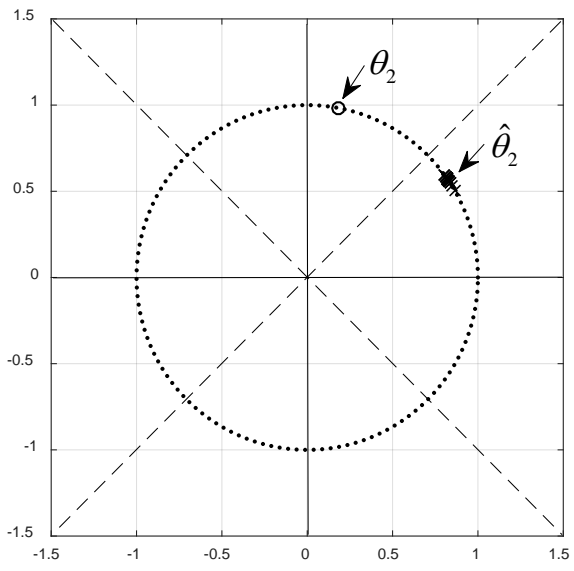

(b)

Fig. 7. Performance of phase offset estimation (a)First-way signal component, (b)Second-way signal component

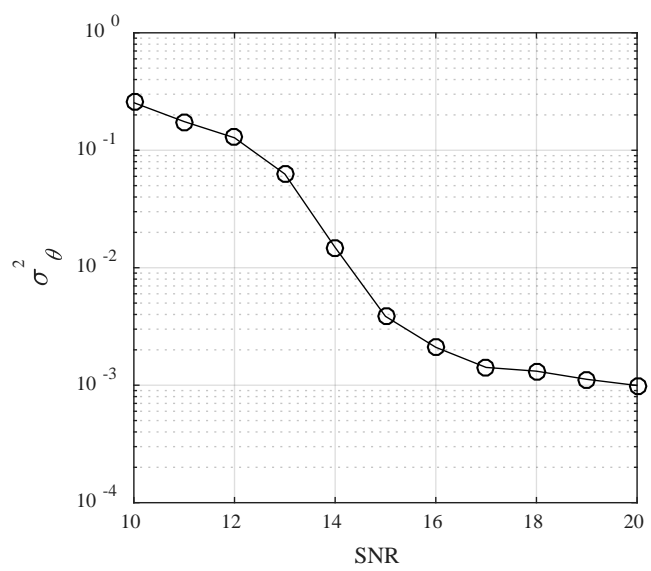

Fig. 8. Performance of phase offset estimation

\subsection{Effect of parameter settings on algorithm performance}

In this section, the time delay is used as the target parameter. Figs. 9 and $\mathbf{1 0}$. show the effect of oversampling factor $N$ and separated symbol length $K$ on algorithm performance. The remaining parameters are the same as those in Section 5.1. As shown in Fig. 9, an obvious improvement in the performance of the algorithm is observed when the oversampling factor increases from $N=1$ to $N=2$ (the estimation variance is reduced about one-order of magnitude less than $16 \mathrm{~dB}$ ), and increases steadily when $N>2$. Fig. 10 shows that the performance of the algorithm is improved with the increase on the number of separated symbols $K$. 


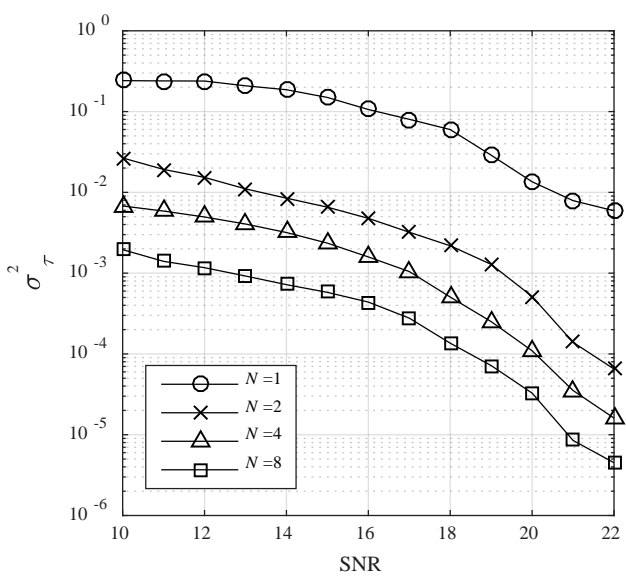

Fig. 9. Effect of $N$ on estimation performance

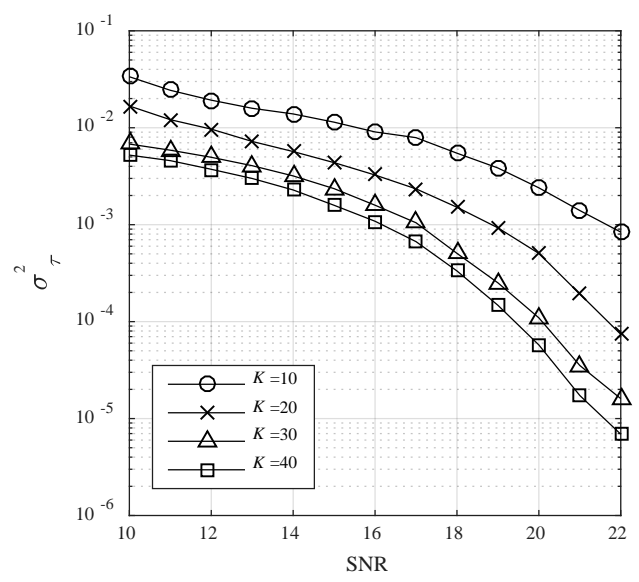

Fig. 10. Effect of $K$ on estimation performance

\section{Conclusion}

In this study, the blind estimation on high-order modulated PCMA signal parameters is realized through the hierarchical searching method based on the derivation of channel stability function. Currently, traditional algorithms have not been able to solve effectively the blind estimation problem of 8PSK modulated PCMA signal parameters without priori information. Compared with the traditional data-aided algorithms, the significant advantage of the proposed method is that no cross-term approximation is performed, leading to a more stable performance. This method does not require any prior information, which meets the requirements of blind processing. In this study, 8PSK modulated PCMA signals are used as an example to examine the algorithm performance, and it can also be extended to other high-order MPSK and QAM modulated PCMA signals.

\section{Acknowledgments}

This work was supported by the National Natural Science Foundation of China (No. 61401511) and the National Natural Science Foundation of China (No. U1736107).

\section{References}

[1] M. Dankberg, "Paired carrier multiple access (PCMA) for satellite communication," in Proc. of Pacific Telecommunications Conference, Honolulu, Hawaii, pp. 787-791, 1998.

Article (CrossRef Link)

[2] F. Li, "Interference cancellation and detection for more than two users," IEEE Transactions on Communication, vol. 59, no. 3, pp. 901-910, 2011. Article (CrossRef Link)

[3] H. Yoshino, K. Fukawa and H. Suzuk, "Interference cancelling equalizer for mobile radio communications,” IEICE Trans. On Communications, vol. 77, no. 4, pp. 849-861, 1997.

Article (CrossRef Link)

[4] C. L. Wu, Z. Liu and X Wang, "Single-channel blind source separation of co-frequency overlapped GMSK signals under constant-modulus constraints," IEEE Communication Letters, vol. 20, no. 3, pp. 486-489, 2016. Article (CrossRef Link) 
[5] Y. Yang, D.L. Zhang andH. Peng. "Frequency Offset Estimation in Single-Channel Linear Mixture of High-Order Modulated Signals,” Acta Electronica Sinica, vol 43, no. 1, pp. 160-165, 2015. Article(CrossRef Link)

[6] X.J. Wei,Y. Yang, Y.M. Guo, et al, "Joint estimation of interference frequency offset and time delay in PCMA system,” Journal on Communications, 38(2), pp. 173-182, 2017. Article(CrossRef Link)

[7] Y. Yang, D. L.Zhang and H.Peng, "Frequency offset estimation of the linear mixture of two co-frequency 8PSK modulated signals,” IET Signal Processing, vol. 9, no. 2, pp. 186-192, 2015. Article (CrossRef Link)

[8] M. Moeneclaey, "On the true and the modified Cramer-Rao bounds for the estimation of scalar parameter in the presence of nuisance parameters," IEEE Transactions on Communication, vol. 46, no. 11, pp. 1536-1544, 1998. Article (CrossRef Link)

[9] H. Steendam and M. Moeneclaey, "Low-SNR limit of the Cramer-Rao bound for estimating the time delay of a PAM, PSK, or QAM Waveform,” IEEE Communication Letters, vol. 5, no. 1,, pp. 31-33, 2001. Article (CrossRef Link)

[10] H. Steendam and M. Moeneclaey , "Low-SNR limit of the Cramer-Rao bound for estimating the carrier phase and frequency of a PAM, PSK, or QAM Waveform,"IEEE Communication Letters, vol. 5, no. 5, pp. 218-220, 2002. Article (CrossRef Link)

[11] S. H.Chen, S. L.Tu and J. Wan, "A sequential Monte Carlo method for blind signal separation using the difference of time delays,” in Proc. of IEEE Region 10 Conference, Taipei, China, 2007. Article(CrossRef Link)

[12] S. L. Tu, S. H. Chen and H.Zheng, "Particle filtering based single-channel blind separation of co-frequency MPSK signals,” in Proc. of International Symposium on Intelligent Signal Processing and Communication Systems, Xiamen, China, pp. 89-92, 2007. Article(CrossRef Link)

[13] C. H. Liao, S. D. Zhou and Z. L. Zhu, "ML-based joint timing estimation algorithm for co-frequency signals,” Systems Engineering and Electronics, vol. 32, no. 6, pp. 1121-1124, 2010. Article(CrossRef Link)

[14] J. Wan, S. L. Tu and C. H. Liao, "Theory and technology on blind source separation of communication signals,” Beijing: National Defense Industry Press, 2012.

[15] T Maksymyuk, L Han and X Ge, “Quasi-quadrature Modulation Method for Power Efficient Video Transmission over LTE Networks,” IEEE Transactions on Vehicular Technology, Vol.63, No.5, pp.2083-2092, June 2014. Article (CrossRef Link)

[16] S. L.Tu, H.Zheng and N.Gu, "Single-channel blind separation of two QPSK signals using per-survivor processing," in Proc. of IEEE Asia Pacific Conference on Circuits and Systems, China, pp.473-476, 2008. Article(CrossRef Link)

[17] X. D.Zhang, “Modern signal processing(second edition)," 2nd Edition,TSING HUA University Press,Beijing, 2002.

[18] Y. Yang, H. Peng and D.L. Zhang, "Modified Cramer-Rao bound of parameter estimation for single-channel mixtures of adjacent-frequency signals,” IET Signal Processing, vol 10, no. 9, pp. 1082-1088, 2016. Article (CrossRef Link)

[19] A. A. Nasir, S. Durrani andR. A. Kennedy, "Particle filters for joint timing and carrier estimation: improved resampling guidelines and weighted Bayesian Cramer-Rao bounds," IEEE Trans. Commun., vol 60, no. 5, pp. 1407-1419, 2012. Article (CrossRef Link)

[20] C. Fritsche, A. Klein and F. Gustafsson, "Bayesian Cramer-Rao bound for mobile terminal tracking in mixed LOS/NLOS environments," IEEE Wire. Commun. Lett., vol 2, no. 3, pp. 335-338, 2013. Article (CrossRef Link) 


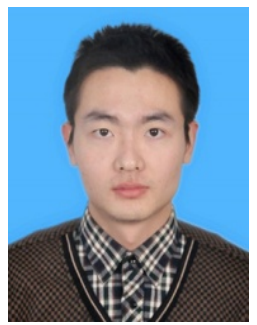

Guo Yiming, male, born in 1990, Ph.D candidate of The China National Digital Switching System Engineering and Technological Research Center, research interests: communication and singal processing, reverse anaysis of signals.

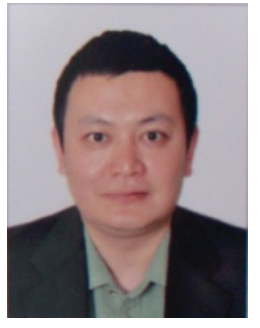

Peng Hua, male, born in 1973, Professor and Ph.D supervisor of The China National Digital Switching System Engineering and Technological Research Center, research interests: Software-defined radio(SDR), communication and singal processing.

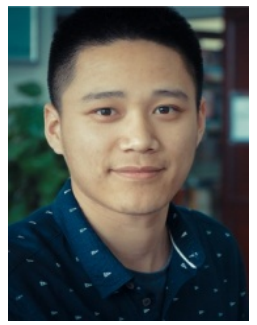

Fu Jun, male, born in 1995, Master of The China National Digital Switching System Engineering and Technological Research Center, research interests: communication and singal processing. 